\title{
INVERTED INTERNAL LIMITING MEMBRANE PEELING TECHNIQUE WITH SILICONE OIL TAMPONADE FOR REPAIR OF RECURRENT LARGE MACULAR HOLES IN VITRECTOMIZED DIABETIC PATIENTS
}

\author{
Ilian Shandurkov, Petja Vassileva \\ Eye Hospital "Prof. Pashev", Sofia, Bulgaria
}

\begin{abstract}
PURPOSE: To present a new approach to treatment of large macular holes in already vitrectomized eyes of patients with proliferative diabetic retinopathy, whose ILM has not been removed during primary surgery.

PATIENTS AND METHODS: Two eyes of two different patients with proliferative diabetic retinopathy which had undergone vitrectomy with endolaser 1.5 to 2 years before admittance to our department. No data for ILM peeling during surgery were available. Our OCT examinations showed macular holes with upper diameter of $765 \mu \mathrm{m}$ in the first patient and $1160 \mu \mathrm{m}$ in the second one. In both cases we combined two well-known surgical approaches: first, inverted ILM flap technique; second, long-term silicone oil tamponade for a period of 4 months.

RESULTS: The macula of both patients was followed up by photographs and regular OCT-s on day one, month one and three months after silicone oil removal. The macular holes remained closed after removal of the tamponade agent. BCVA was 0.5 three months after the last surgery in both patients. During the final examination, macular contour seemed almost normal.

DISCUSSION: Large, long-standing macular holes are less likely to be closed after pars plana vitrectomy. The inverted ILM flap technique has been recently reported to be successful in nearly $99 \%$ of patients with macular holes more than $400 \mu \mathrm{m}$ in diameter. The position of the inverted ILM flap is much more controllable under silicone oil than under gas tamponade.
\end{abstract}

Keywords: inverted ILM peeling, silicone oil tamponade, recurrent macular holes

Address for correspondence:

Ilian Shandurkov, MD, PhD

Eye Hospital "Prof. Pashev"

Sofia, Bulgaria

e-mail: ilian_sh@yahoo.com

Received: October 16, 2014

Accepted: December 19, 2014

\section{INTRODUCION}

Macular hole (MH) is a macular disease affecting mostly women after their $5^{\text {th }}$ decade. It could be idiopathic or associated with other ocular conditions such as diabetic retinopathy, peripheral retinal breaks, uveitis, retinal vein occlusion or extensive laser photocoagulation in the past.

Prior to 1991, the disease was thought to be untreatable until Kelly and Wendel (1) reported 58\% success rate after pars plana vitrectomy. Nowadays, due to new surgical equipment and techniques, the 
success rate after macular hole surgery is reported to be over $85 \%$ (2).

The diagnosis and understanding of the formation of macular holes $(\mathrm{MH})$ have greatly benefited from examination by optical coherence tomography (OCT), which indeed allows easy differentiation between full-thickness $\mathrm{MH}$ and a pseudohole (3). OCT also facilitates measuring the diameter of the hole, which is an important prognostic factor. The measurement is mandatory from the surgical point of view, as different techniques could be applied to achieve closure of $\mathrm{MH}$.

For large macular holes, different techniques have been proposed to improve surgical outcome $(4,5)$. Recently, a prospective randomized study proved that the "inverted internal limiting membrane flap (inverted ILM flap) technique" improves functional and anatomic results in patients with large macular holes as compared to pars plana vitrectomy with ILM peeling and air tamponade (6). The question "How to treat already vitrectomized eyes with large macular holes more than $400 \mu \mathrm{m}$ in diametre" does not have a unanimous answer yet.

\section{PURPOSE}

To present a new approach to treatment of large macular holes in already vitrectomized eyes of patients with proliferative diabetic retinopathy, whose ILM has not been removed during primary surgery.

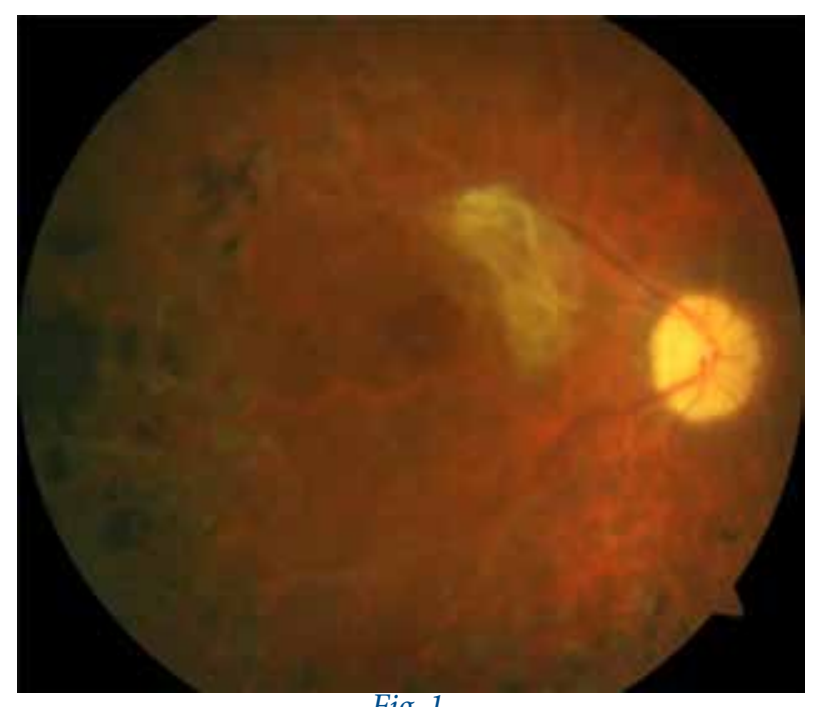

Fig. 1.

\section{PATIENTS AND METHODS}

Two eyes of two different patients with proliferative diabetic retinopathy were operated for vitreous haemorrhage. Both patients had type I Diabetes mellitus and had been on permanent Insulin treatment for more than 20 years. They had undergone vitrectomy with endolaser 1.5 to 2 years before admittance to our department. No data for ILM peeling during surgery were available.

Our OCT examinations showed macular holes with upper diameter of $765 \mu \mathrm{m}$ in the first patient and $1160 \mu \mathrm{m}$ in the second one.

Case 1: a 38-year old female who has suffered from Diabetes mellitus type I for 22 years. She has been on Insulin treatment since the establishment of diagnosis. Over the last 10 years she has had multiple laser photocoagulation sessions. Despite the relatively good glycemic control, she developed a proliferative diabetic retinopathy. Both eyes had been vitrectomized for vitreous haemorrhages two years before admittance to our department. Our clinical examination found a large macular hole in the right eye with concomitant epiretinal fibrotic tissue over the nerve fiber layer in the area between the optic nerve head and the macula (Fig. 1). The upper diameter of the hole was $765 \mu \mathrm{m}$ (Fig. 2). The fellow eye had diabetic ischemic optic neuropathy and very low visual acuity. Best corrected visual acuity (BCVA) of the right eye was 0.1 and BCVA of the left eye -0.05 . Both eyes were pseudophakic for more than 3 years.

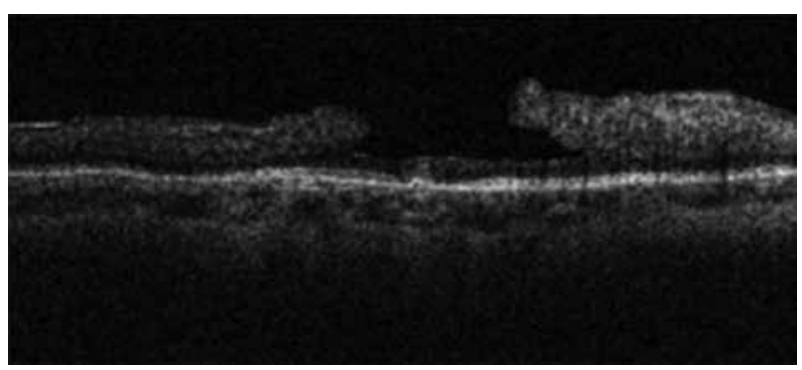

Fig. 2.

Case 2: a 64-year old male with history of Diabetes mellitus type I for over 35 years. He had been on Insulin treatment from the beginning, but there were periods of poor control of the disease. The patient's right eye was treated only with laser and has a mild cataract. The fellow left eye had been vitrec- 
tomized for vitreous haemorrhage 1.5 years before. Since then the visual acuity had been gradually decreasing and the images were strongly distorted. He developed a nuclear cataract and a large macular hole (Fig. 3). The upper diameter of the hole was $1160 \mu \mathrm{m}$ (Fig. 4).

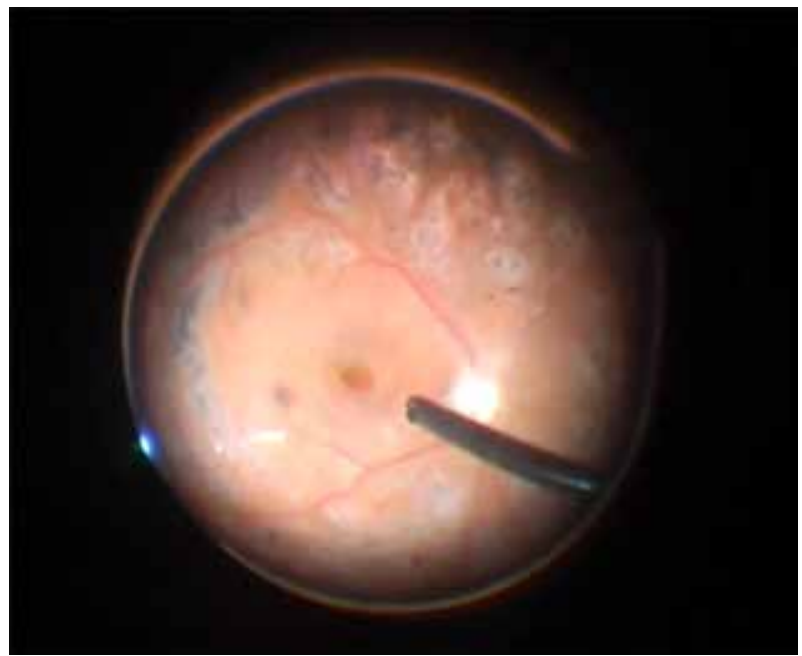

Fig. 3. Case 2 surgery

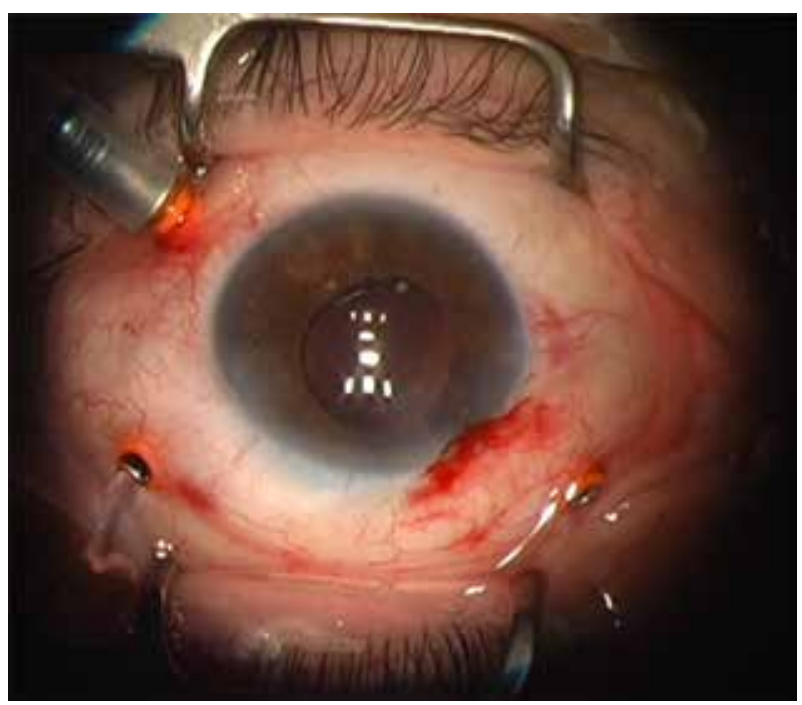

Fig. 5.

Best corrected visual acuity (BCVA) of the right eye was 0.8 because of the initial cataract, and BCVA of the left eye -0.02 due to combination of cataract and macular hole.

Surgical technique: In both cases we combined two well-known surgical approaches: first, inverted
ILM flap technique with thorough drying of the posterior pole; second, long-term silicone oil tamponade for a period of 4 months.

Our technique consisted of phacoemulsification and implantation of hydrophobic acrylic monoblock lens in the second patient with cataract as first

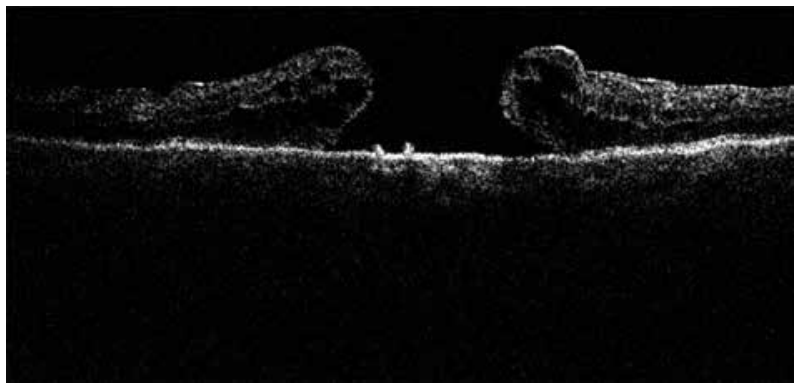

Fig. 4.

step. Next, both surgeries were made in the same manner. We used $23 \mathrm{Ga}$ transconjunctival sutureless approach to the retina (Fig. 5). As vitreous was previously removed, we made Brilliant blue G-assisted ILM peeling (Fig. 6).

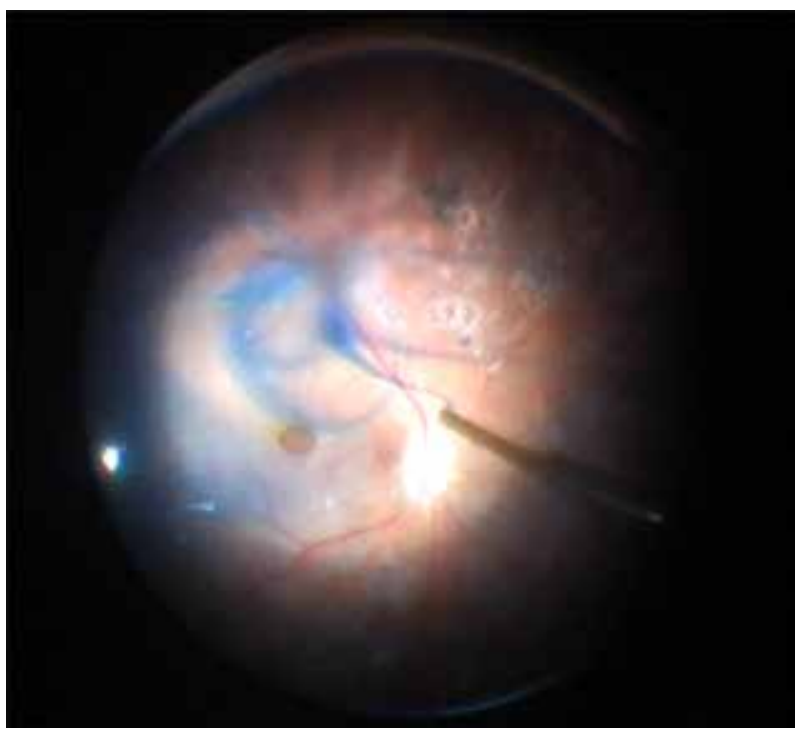

Fig. 6.

During the circumferential peeling, the ILM was not removed completely from the retina but was left attached to the edges of the macular hole. A rolled segment of the peeled ILM was hanging in the vitreous cavity. Next, a peripheral piece of the ILM was trimmed with a vitreous cutter and the central part 
of the ILM was left in place. As a result, small remnants of the ILM remained surrounding the macular hole. The ILM was then gently massaged into the macular hole from all sides until the ILM became inverted, or upside down, so that the surface which normally faces the vitreous body now faced the retinal pigment epithelium. The macular hole was covered with the inverted ILM flap. The next step was to perform fluid-air exchange with thorough drying of the posterior pole. To that end, we used 25Ga backflush cannula with low levels of intraocular pressure controlled by the machine. At the end of the surgery, the vitreous cavity was filled with silicone oil and the pressure was left to $10 \mathrm{mmHg}$.

The patients were advised to maintain a facedown position for one week, although we observed closure of the holes on the operating table.

\section{RESULTS}

The macula of both patients was followed up by photographs and regular OCT-s on day one, month one and three months after silicone oil removal (Fig. 7-10).

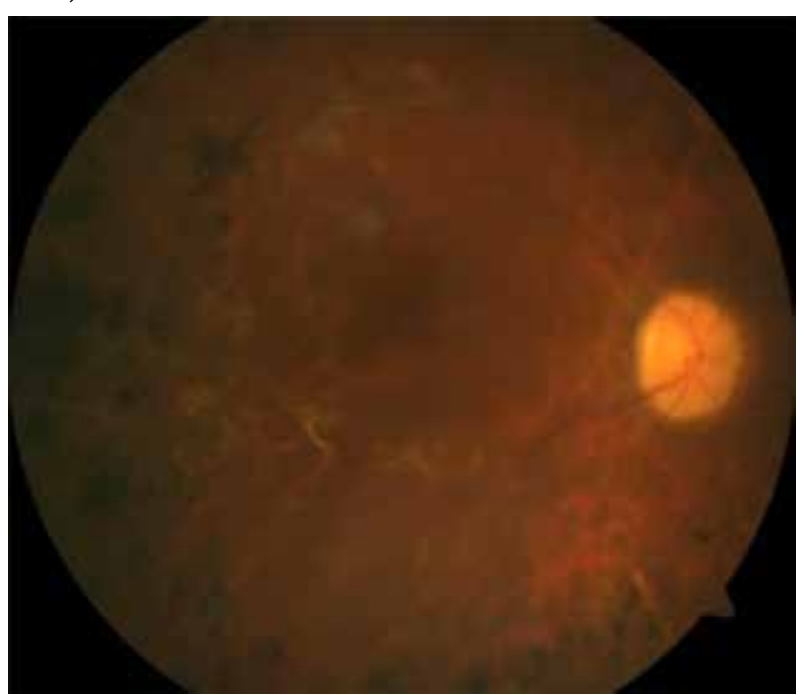

Fig. 7. Case 1

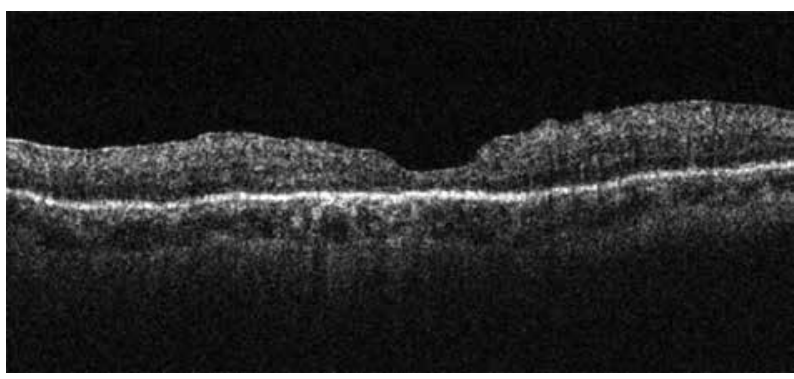

Fig. 8. Case 1

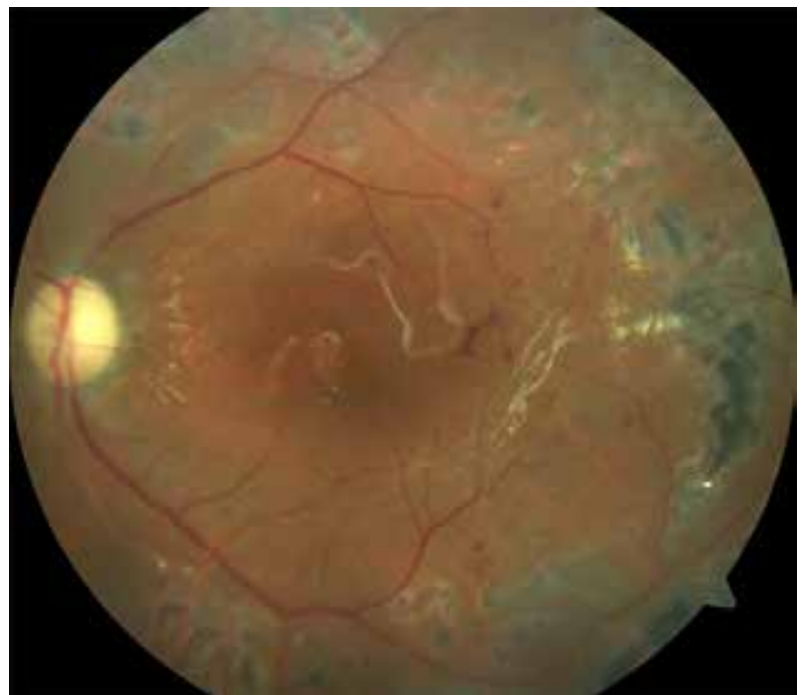

Fig. 9. Case 2

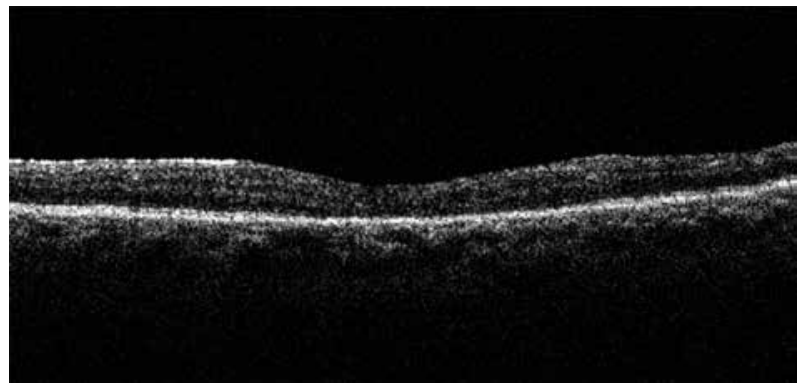

Fig. 10. Case 2

The macular holes remained closed after removal of the tamponade agent. BCVA was 0.5 three months after the last surgery in both patients. During the final examination, the macular contour seemed almost normal (Fig. 11, 12). Small defects of the junction between outer and inner segments of the photoreceptors and external limiting membrane remained paracentral to the fovea. On the inner retinal surface, a delicate hyperreflective structure can be seen without any evidence of epiretinal membrane formation.

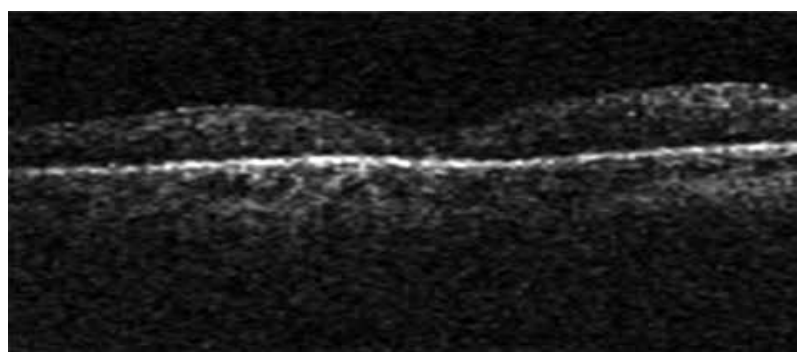

Fig. 11. Case 1 


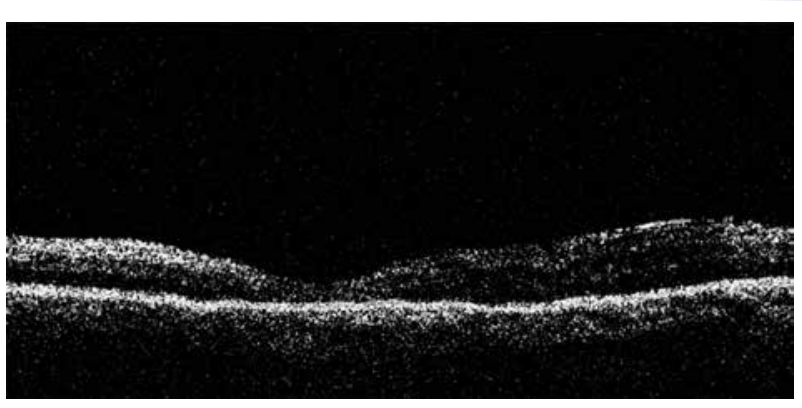

Fig. 12. Case 2

\section{DISCUSSION}

The ultimate goal of macular hole surgery is visual improvement. Since improvement in postoperative vision has been proved to be related to the anatomical closure of the hole, surgeons and researchers have concentrated their efforts on modifying their surgical technique to achieve a better hole closure rate.

Large, long-standing macular holes are less likely to be closed after pars plana vitrectomy. Extensive laser treatment to the retina combined with epiretinal proliferations due to proliferative diabetic retinopathy are factors worsening the prognosis of preexisting macular holes.

The inverted ILM flap technique has been recently reported to be successful in nearly $99 \%$ of patients with macular holes more than $400 \mu \mathrm{m}$ in diameter (6). Failures were observed in eyes in which it was impossible to perform the inverted ILM technique due to the fact that the ILM had been previously removed. In such cases, long-term tamponade with silicone oil leads to additional force for proper adhesion of free margins of the macular hole.

The position of the inverted ILM flap is much more controllable under silicone oil than under gas tamponade. On the other hand, the thorough drying of the macula after the fluid air exchange helps adequate retinal pigment epithelium pump function and respectively the sufficient adhesion with the neurosensory retina.

It was documented by OCT that, in most cases, macular holes close in the first days after surgery (7). However, in some cases the closure process is longer, especially in large macular holes (8).

Postoperative positioning of the head depends on the desired duration of the tamponade on the macula. Two randomized series which studied the face-down positioning have been performed (9). One showed a significant drop in the success rate without face-down positioning, but a post hoc analysis suggested that this reduction was only significant for macular holes greater than $400 \mu \mathrm{m}$ and not for smaller holes $(10,11)$.

In this paper, we present our approach consisting of use of silicone oil tamponade as an adjunct to the inverted flap technique in patients with already vitrectomized eyes, but without ILM peeling during primary surgery. It may be observed that beneath the silicone oil, the fragments of ILM cover the macular defect.

To conclude, this report suggests that postoperative prone positioning is an important condition for closure of large macular holes. Silicone oil can be used in separate cases as an adjunct to the inverted ILM flap technique. Also, postoperative improvement of visual acuity and retinal architecture may last many months after silicone oil removal in macular hole surgery.

\section{REFERENCES}

1. Kelly NE, Wendel RT. Vitreous surgery for idiopathic macular holes: results of a pilot study. Arch Ophthalmol, 1991;109:654-659.

2. Beutel J, Dahmen G, Ziegler A, Hoerauf H. Internal limiting membrane peeling with indocyanine green or trypan blue in macular hole surgery: a randomized trial. Arch Ophthalmol, 2007;125:326-332.

3. Hee MR, Puliafito CA, Wong C, et al. Optical coherence tomography of macular holes. Ophthalmology, 1995;102:748-56.

4. Alpatov S, Shchuko A, Malyshev V. A new method of treating macular holes. Eur J Ophthalmol, 2007;17:246-252.

5. Couvillion SS, Smiddy WE, Flynn HW, Jr, Eifrig CWG, Gregori G. Outcome of surgery for idiopathic macular hole: a case-control study comparing silicone oil with gas tamponade. Ophthalmic Surg Lasers Imaging, 2005;36:365-371.

6. Michalewska Z, Michalewski J, Adelman RA, Nawrocki J. Inverted internal limiting membrane (ILM) flap technique for large macular hole. Ophthalmology, 2010;117:2018-2025.

7. Jumper JM, Gallemore RP, McCuen BW, 2nd, Toth CA. Features of macular hole closure in the ear- 
ly postoperative period using optical coherence tomography. Retina, 2000;20:232-237.

8. Michalewska Z, Michalewski J, Nawrocki J. Continuous changes in macular morphology after macular hole closure visualized with spectral optical coherence tomography. Graefes Arch Clin Exp Ophthalmol, 2010;248:1249-1255.

9. Eckardt C, Eckert T, Eckardt U, et al. Macular hole surgery with air tamponade and optical coherence tomography-based duration of face-down positioning. Retina, 2008; 28:1087-96.

10. Guillaubey A, Malvitte L, Lafontaine PO, et al. Comparison of face-down and seated position after idiopathic macular hole surgery: a randomized clinical trial. Am J Ophthalmol, 2008; 146:128-34.

11. Tadayoni R, Vicaut E, Devin F, et al. A randomized controlled trial of alleviated positioning after small macular hole surgery. Ophthalmology, 2011;118:150-5. 